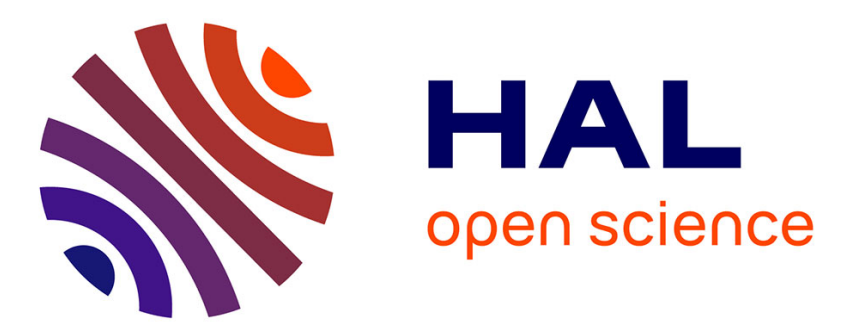

\title{
A three-dimensional Dionne model for multipactor simulations
}

\author{
Adrien Plaçais, Mohamed Belhaj, Julien Hillairet, Jérôme Puech
}

\section{To cite this version:}

Adrien Plaçais, Mohamed Belhaj, Julien Hillairet, Jérôme Puech. A three-dimensional Dionne model for multipactor simulations. Physics of Plasmas, inPress, 27 (5), pp.053512. 10.1063/5.0004076 . hal-02472458v4

\section{HAL Id: hal-02472458 \\ https://hal.science/hal-02472458v4}

Submitted on 28 May 2020

HAL is a multi-disciplinary open access archive for the deposit and dissemination of scientific research documents, whether they are published or not. The documents may come from teaching and research institutions in France or abroad, or from public or private research centers.
L'archive ouverte pluridisciplinaire HAL, est destinée au dépôt et à la diffusion de documents scientifiques de niveau recherche, publiés ou non, émanant des établissements d'enseignement et de recherche français ou étrangers, des laboratoires publics ou privés. 


\title{
A three-dimensional Dionne model for multipactor simulations
}

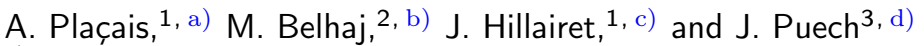 \\ ${ }^{1)}$ CEA, IRFM, F-13108 Saint-Paul-Lez-Durance, France \\ 2) DPHY, ONERA, Université de Toulouse F-31055 Toulouse, France \\ 3) CNES, DSO/RF/HNO, F-31401 Toulouse, France
}

The multipactor phenomenon is characterized by a very fast growth of the electronic population in RadioFrequency ( $R F$ ) devices under vacuum. As this effect limits the transmissible RF power and can harm RF systems, it has been widely studied during the last decades. Due to the high cost of experimental tests, simulation tools are heavily used to predict the threshold of multipactor growth. However, their reliability is limited for complex configurations, e.g. when dielectrics or magnetic fields are present. A crucial element of these multipactor simulations is the secondary-emission model. Dionne's model is able to model both metals and dielectrics secondary emission but is one-dimension only. As the three-dimensional aspect is essential for complex configurations, the Dionne model is extended do three-dimensions. Measurements of the total electron emission yield have been carried out at the ONERA and shows a good agreement for low-impact energy and low-impact angle electrons, which is relevant in multipactor simulations.

\section{INTRODUCTION}

The multipactor effect is an electronic avalanche that may appear in vacuum RF systems when two conditions are met: (i) electrons enter in resonance with the $\mathrm{RF}$ field and (ii) their energy is sufficiently high to extract additional electrons from the system walls. In practice, multipactor appears when a RF electric field threshold $\mathbf{E}_{\max }$ is reached. This phenomenon has been observed in telecommunications satellites ${ }^{1}$ and in RF plasma heating systems of experimental fusion reactors (tokamaks) ${ }^{2}$, but also in particle accelerators ${ }^{3}$. The multipactor creates an electron cloud, leading to a disturbance or even a reflection to the source of RF signal. A breakdown can also be created, leading to a rapid heating of the surfaces, an alteration of the system material's properties or an out-gassing triggering the destructive corona effect ${ }^{4}$.

As multipactor threshold measurements are expensive, alternative methods have been developed to estimate it: theoretical $^{5,6}$ and statistical ${ }^{7}$ studies, Monte-Carlo ${ }^{8}$ or Particle-in-Cell (PIC) simulations ${ }^{9,10}$. A PIC code has been developed in Refs. 11-13 in order to understand the apparition of this phenomenon in complex RF devices from a physical point of view. This approach requires an accurate modelling of the physics at stake, and especially of the electron emission phenomenon.

Let us consider a sample impacted by a flux of electrons, called Primary Electrons (PEs). A second flux of electrons may be emitted by the sample; it is made up of Secondary Electrons (SEs, which are electrons removed from the material), of Inelastically and Elastically Backscattered Electrons (IBEs and EBEs, which are PEs rediffused by the sample respectively with and without

\footnotetext{
a) Electronic mail: adrien.placais@cea.fr; also at DPHY, ONERA, Université de Toulouse F-31055 Toulouse, France; CNES, DSO/RF/HNO, F-31401 Toulouse, France

b) Electronic mail: mohamed.belhaj@onera.fr

c) Electronic mail: julien.hillairet@cea.fr

d) Electronic mail: jerome.puech@cnes.fr
}

energy loss). The Secondary Electron Emission Yield $(\delta$, SEEY) is defined as the ratio between the SEs and PEs fluxes. It is higher than one if there are more SEs than PEs. The Elastically Backscattered Electron Emission Yield $\left(\eta_{e}\right.$, EBEEY) and Inelastically Backscattered Electron Emission Yield $\left(\eta_{i}\right.$, IBEEY) are similarly defined. Finally, the Total Electron Emission Yield $(\sigma$, TEEY) is the ratio between all emitted electrons and PEs fluxes. $\sigma>1$ is a necessary condition for the electron population to grow and thus for the multipactor to appear. We have the relation:

$$
\sigma=\delta+\eta_{e}+\eta_{i}
$$

A full modelling of the three emitted electrons populations require the knowledge of their emission yields, as well as their energies and angular distributions. All these quantities rely on the energy and direction of the PEs flux ${ }^{14}$, on the surface morphology of the sample ${ }^{15}$, on the presence of contaminants ${ }^{16}$ or external magnetic fields ${ }^{17}$. In addition, dielectric materials, which are widely used in spacecraft applications, can hold a net electric charge. It influences electron emission through two processes: firstly, the created surface potential may have an effect on electrons trajectories in the vacuum. Secondly, the excess of holes or electrons inside the material modifies the transport of electrons when they are in the sample. SEs are the population most influenced as they are the least energetic.

As SEs are the predominant population in waveguides, the SEEY mostly conditions the multipactor apparition. SEEY models exist ${ }^{18-20}$, but, to our knowledge, the Dionne model $^{21}$ is the only one able to take into account the influence of dielectric charge on the transport of SEs inside the material ${ }^{22}$ while being sufficiently simple for PIC simulations, which involve large numerical resources. Another asset of Dionne's model is that it gives $\delta$, while some other models only give $\sigma^{23-25}$. Thus, Dionne's model allows SEs to be discriminated from backscattered electrons. EBEs have been shown to be of great importance in multipactor apparition ${ }^{26}$, especially when magnetic fields are present ${ }^{27}$. EBEs were also shown to be 
significant in Hall plasma thrusters energy balance ${ }^{28}$.

The main limitation of Dionne's model is that it is one-dimension only and cannot take into account for the incidence angle of PEs; this restriction becomes a limitation when simulating complex $3 \mathrm{D}$ geometries such as circulators. Circulators are used to avoid RF power reflection to the source, that would cause it's degradation. The central part of circulators is composed of ferrites that may have a dielectric behavior; they also create a static magnetic field parallel to the RF electric field, reason why a three-dimension simulation becomes mandatory. A preliminary study has been published ${ }^{13}$. We present here the complete study providing detailed explanations, comparison of the model on materials with various surface treatments and the introduction of the equivalent incidence angle notion.

In this paper, we develop a three-dimension version of Dionne's model for such configurations. In section II, we make a simple simulation to evaluate the validity range relatable for multipactor study. In section III, we develop the secondary emission model. Finally, we benchmark it with in-house electron emission yield measurements in section IV.

\section{MODEL FRAMEWORK}

We define here the validity range of the proposed SEEY model in terms of PEs' impact energy and incidence angle. Preliminary multipactor simulations have been made with the simulation code POTOMAC (Physical simulatiOn TOol for Multipactor in Advanced Configurations) ${ }^{11-13}$. POTOMAC is a PIC code that computes the evolution of the electron population in a domain with a given RF electric field. Each time a collision occurs, $\delta, \eta_{e}$ and $\eta_{i}$ are calculated and the appropriate emission mechanism is chosen. Here, the multipactor threshold was computed for a WR75 rectangular waveguide with silver coated walls. The RF signal has a frequency of $11 \mathrm{GHz}$ and propagates in the $\mathrm{TE}_{1,0}$ mode, which is the fundamental mode for this geometry. Dimensions are $19.05 \mathrm{~mm} \times 9.525 \mathrm{~mm}$. The emission yield, angular emission distribution and energy emission distribution for the three populations are listed in Tab. I. All data calculated with the Monte-Carlo code $\mathrm{SLAB}^{29}$ were tabulated in order to reduce the computational time.

\begin{tabular}{lccc}
\hline & Emission Yield & Angular distrib. & Energy distrib. \\
\hline SE & Dionne (this paper) & Cosine law $^{30}$ & Chung et al. ${ }^{31}$ \\
EBE & SLAB $^{29}$ & SLAB $^{29}$ & Monoenergetic \\
IBE & Constant $^{30}$ & Cosine law $^{30}$ & Uniform \\
\hline
\end{tabular}

TABLE I: Full electron emission model used with POTOMAC for the preliminary simulation

We recorded the impact energy of all PEs at the multipactor threshold and represented it as an histogram in Fig. 1. Seed Electrons are the electrons injected in the waveguide during the first periods of the simulation. These results first show that SEs are predominant in POTOMAC simulations, and thus must be modelled with great attention. Secondly, $99 \%$ of PEs impact the walls with an energy lower than $230 \mathrm{eV}$. In order to take into account for the various configurations, the model should be valid up to $300 \mathrm{eV}$.

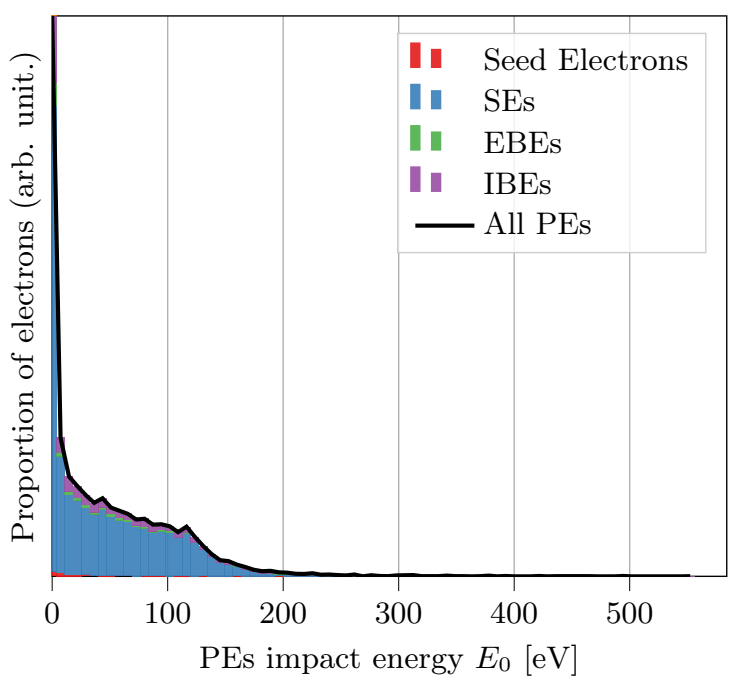

FIG. 1: Histogram of the PEs impact energy

Fig. 2 is a histogram of the SEs angular flux when they impact a wall $\theta_{0} / \sin \theta_{0}, \theta_{0}$ being defined relative to the wall normal (see Fig. 3). It shows us that the majority of SEs impact surfaces almost perpendicularly; as we want our model to be usable in the broadest variety of scenarios possible, it should be valid for angles ranging from $0^{\circ}$ to $40^{\circ}$.

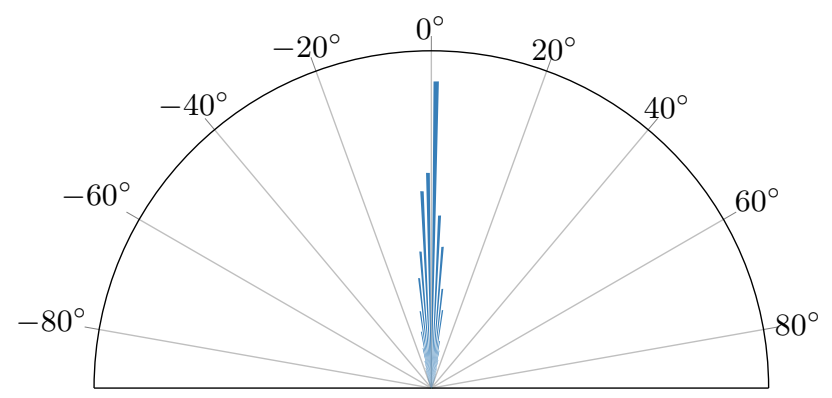

FIG. 2: Histogram of the flux of impacting SEs according to their collision angle $\theta_{0}$

This basic configuration was chosen for the sake of simplicity, and for that it is representative. Using POTOMAC, we saw that in waveguides with smaller gaps, electrons tended to impact walls with a smaller energy. The distribution of impact angle was slightly more spread around the central value of $0^{\circ}$. On the contrary, in waveguides with greater gaps, the impacting energy of electrons 
rose. This is not a problem, given that multipactor generally occurs where the gap is small and the electric field high. We also found that adding magnetic fields or dielectrics with a low surface charge did not change the order of magnitude of PEs' impact energy and incidence angle. In presence of highly charged dielectrics, some electrons can impact walls with energies up to a few $\mathrm{keV}$; however, both the mean and the median of the $E_{0}$ distribution are shifted towards lower energies. This $E_{0}$ and $\theta_{0}$ study should be led again for more complex geometries. We do not see any reason that a significant fraction of electrons impacted surfaces with energies higher than $300 \mathrm{eV}$. In the contrary, the $\theta_{0}$ distribution may be severely distorted towards extreme angles. This could be the case in iris for example.

Most RF systems use copper or silver, which have comparable emission properties. The nature of the material do not influence much $E_{0}$ nor $\theta_{0}$. However, very poor emitting materials would drastically increase the electric field threshold $\mathbf{E}_{\max }$, which would in consequence increase $E_{0}$.

The model we propose can be used in other fields than multipactor, as long as electrons impact surfaces with a low energy and a low incidence angle.

\section{SEEY MODEL}

\section{A. Dionne in 1D}

Numerous secondary emission models already exist; for example, Monte-Carlo methods are precise and can take into account all the physical processes involved, but are very slow, which is prohibitive for PIC simulations. On the other side of the spectrum, very fast and simple models exist, such as Vaughan ${ }^{23,24}$. This model is $3 \mathrm{D}$ and can take into account the materials rugosity. However, it has the inconvenience of returning a null TEEY for very low energy PEs, which constitutes an important proportion of impacting electrons (see Fig. 1) and is not consistent with measurements ${ }^{32}$. Plus, this model cannot discriminate SEs from IBEs and EBEs.

A modified version of Vaughan has been proposed ${ }^{33}$, which manually forces a TEEY equal to unity for lowenergy electrons; it also fits a parameter $E_{\text {threshold }}$ to enforce the model to match $\sigma\left(E_{c 1}\right) . \quad E_{c 1}$ is the first cross-over energy, defined as the first energy to verify $\sigma\left(E_{c 1}\right)=1$. This point has been shown of being of primordial importance for multipactor simulations ${ }^{34}$. The modified Vaughan model is widely used by the multipactor community.

Dionne's model is a fast, simple and physical model for the SEEY. It makes the assumption that secondary emission process can be divided into three stpdf:

1. a PE enter a sample and emit SEs on its trajectory inside the material;
2. SEs are transported from their emission point to the surface;

3. SEs escape the sample through the surface.

All terms in the Dionne model have a physical meaning. Thus, it is for example possible to analytically reflect the influence thin coatings have on the SEEY and hence on the multipactor ${ }^{12,35}$. However, Dionne's model does not take into account for the incidence angle of the PE, unlike Vaughan.

For the sake of clarity, Dionne's model is explained here. Under the hypothesis of a constant energy loss for the PE and no scattering for the SEs, it may be written as:

$$
\delta=G \cdot T \cdot S
$$

Where the generation term $G$ represents the mean number of SEs produced by the PE, the transmission term $T$ represents the probability for the produced SEs to reach the surface and the escape term $S$ is a sample dependent constant representing the probability for the SEs to cross the surface. This terms can be expressed as:

$$
G=\frac{E_{0}}{\Phi \cdot R\left(E_{0}\right)}
$$

$$
T=d \cdot\left(1-\mathrm{e}^{-R\left(E_{0}\right) / d}\right)
$$

Where $n$ is a material dependent constant, $E_{0}$ is the energy of the $\mathrm{PE}$ and $\Phi$ the material effective work function $^{36}$ defined relative to the vacuum level in $\mathrm{eV}$. For a metal, $\Phi=W_{f}$ with $W_{f}$ the work function and for a dielectric, $\Phi=\chi+E_{g}$ with $\chi$ the electron affinity and $E_{g}$ the gap energy. $R$ is the range of the PE (see Fig. 3) and $d$ the diffusion length of the $\mathrm{PE}$ in the material in $\mathrm{nm}$. Using the continuous slowing down approximation, with $A$ a surface-dependent constant, the range can be expressed as $^{21,37}$ :

$$
R\left(E_{0}\right)=\frac{E_{0}^{n}}{A \cdot n}
$$

$\Phi$ can be measured or calculated ${ }^{38}$. It was suggested that $d$ was equal to the inelastic mean-free path ${ }^{39}$; however, very low energy electrons trajectories are mostly driven by elastic collisions ${ }^{18}$, making this assumption unsuitable for our study. $d$ also can be calculated with the density and the effective absorption coefficient ${ }^{40}$. Thermionic emission experiments allow us to measure $S^{40}$. $A$ and $n$ can be extracted from Monte-Carlo simulations or measurements ${ }^{37}$.

In this study, $\Phi$ will be taken from literature while $A$, $n, d$ and $S$ will be fitted, for that they drastically vary from one sample to another - especially when samples are contaminated. 


\section{B. Dionne in 3D}

At a given energy and if the considered sample is perfectly flat, PEs penetrate less and less deep while $\theta_{0}$ increases (see Fig. 3). Thus, SEs are emitted closer and closer to the surface and are more likely to be emitted in the vacuum, increasing the SEEY.

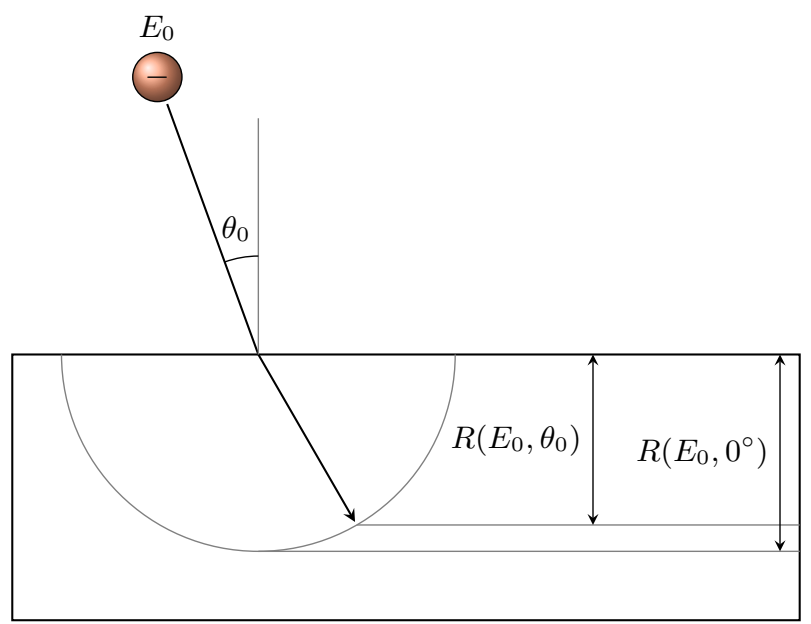

FIG. 3: Macroscopic view of a PE impacting a sample with an energy $E_{0}$ and an angle $\theta_{0}$

The Dionne model was not designed to take the incidence angle $\theta_{0}$ into account. As it is physical, it is however possible to add an angle dependency. Modifying $\theta_{0}$ does not influence the distance travelled by the PE but only its range (see Fig. 3). The Snell's law for electron reads $^{41}$.

$$
\sqrt{E_{0}} \sin \theta_{0}=\sqrt{E_{1}} \sin \theta_{1}
$$

where $E_{1}$ and $\theta_{1}$ are respectively the energy and angle of the electron after it has been refracted in the sample. The range thus can be expressed as:

$$
R\left(E_{0}, \theta_{0}\right)=R\left(E_{0}, 0^{\circ}\right) \sqrt{1-\frac{E_{0}}{E_{0}+\Phi} \cdot \sin ^{2} \theta_{\text {equ }}}
$$

$\theta_{\text {equ }}$ is the equivalent incidence angle. For a perfectly flat sample, $\theta_{\text {equ }}$ can be replaced by $\theta_{0}$. For any other material, $\theta_{\text {equ }}$ is a function of $\theta_{0}$ and of the sample rugosity profile. It firstly takes into account the fact that the macroscopic angle $\theta_{0}$ is different from the microscopic impact angle, due to the surface roughness (oblique incidence effect). Other phenomenons are taken into account: if the sample presents a small roughness, lowenergy high incidence angle electrons that could not be emitted on a flat surface can escape an inclined plane, increasing the SEEY at low energy ${ }^{15}$. For high roughness materials, SEs are more likely to be recollected by the sample, which causes a reduction of the SEEY (shading effect $)^{15}$. This effect is more pronounced in presence of magnetic fields ${ }^{17}$. Recollected SEs can also emit other electrons (multigeneration).
Zhang et $a .^{42}$ showed with Monte-Carlo simulations that it was possible to replace $\theta_{0}$ with $\theta_{\text {equ }}$ in TEEY calculations to take all these effects into account, for random rough surfaces with Gaussian height distribution. Their results suggested that a first order approximation could be:

$$
\theta_{\text {equ }}\left(\theta_{0}\right)=k_{a} \theta_{0}+k_{b}
$$

With $k_{a}$ and $k_{b}$ rugosity profile dependent constants. For a perfectly flat material, $\theta_{\text {equ }}=\theta_{0}$ which implies $k_{a}=1$ and $k_{b}=0^{\circ}$

\section{MODEL VERIFICATION}

\section{A. Experimental procedure}

The TEEY measurements were performed in an Ultra High Vacuum (UHV) facility designed for the study of electron emission. A dry turbo-molecular pump associated with an oil-free primary pump allows the system to be maintained at a vacuum level down to $3 \times 10^{-9}$ mbar. The sample holder allows the variation of the electron incidence angle from $0^{\circ}$ (normal incidence angle) to $80^{\circ}$. An ELG-2022B electron gun from Kimball Instrument was used. The electron beam was pulsed during TEEY measurements to limit the surface conditioning effect ${ }^{43}$. The facility is equipped with a Faraday cup, a hemispherical electron energy analyser and a X-ray Mgkalpha source. The sample holder and the collector can be independently biased to choose the desired potential. The sample holder is ordinarily negatively biased in order to prevent the low energy emitted electron to come back to the sample surface and prevent the generation of tertiary electrons.

TEEY measurements were made on several samples [Associated dataset available at http://dx.doi.org/ 10.5281/zenodo.3648704] (Ref. 44). In this paper, we decided to compare the developed model to copper only, which is often used in RF components. We did so to facilitate the comparison between samples and to focus on their emission properties evolution with surface treatment.

The first sample, called hereafter $\mathrm{Cu} \# 1$, was heated in situ and under UHV at $200{ }^{\circ} \mathrm{C}$ for $2 \mathrm{~h}$. A second set of measurements was made after $3 \mathrm{~h}$ of in situ erosion under $1 \mathrm{keV} \mathrm{Ar}^{+}$flux at normal incidence. XPS study showed that after the heating, the sample was contaminated, mainly with oxygen and carbon; after the erosion, it was almost pure.

A second set of measurements was made on another copper sample, $\mathrm{Cu} \# 2$. Measurements were made on the sample without any treatment (as received), after $2 \mathrm{~h}$ of heating at $200{ }^{\circ} \mathrm{C}$ in situ and under UHV, and after $1 \mathrm{~h}$ of in situ erosion under $1 \mathrm{keV} \mathrm{Ar}^{+}$flux at normal incidence. 


\section{B. Fitting process}

The total backscattered electron emission yield $\eta$ is defined as:

$$
\eta=\eta_{e}+\eta_{i}
$$

We computed $\eta$ with the Monte-Carlo software CASINO v3.3.0.4 $4^{45}$. ELSEPA model was used for the total and partial cross-section calculation ${ }^{46}$, the direction cosines were calculated with Lowney et al. ${ }^{47}$, the stopping power and ionisation potential with Joy et $a l^{48}$. The pseudorandom number generator was a Lagged Fibonacci generator. Results showed a very weak dependence of $\eta$ on $\theta_{0}$.

We computed $\eta_{e}$ with the SLAB model ${ }^{29}$. Due to the lack of literature on the subject, we considered that $\eta_{i}$ was a constant, independent from $E_{0}$ and $\theta_{0}$. As our values of $\eta$ and $\eta_{e}$ are accurate for $E_{0}=500 \mathrm{eV}$ and $\theta_{0}=0^{\circ}$, we set $\eta_{i}$ so that Eq. (9) holds at $500 \mathrm{eV}$ and $0^{\circ}$. The Dionne model was then fitted on measurements with $E_{0}$ in $0-300 \mathrm{eV}$ and $\theta_{0}$ in $0^{\circ}-40^{\circ}$.

\section{Evaluation of the model's capacity to fit $\sigma$}

The Dionne model was compared to modified Vaughan model as defined in Ref. 33. For almost all materials, Vaughan rugosity parameters $k_{E}$ and $k_{\theta}$ should be equal to unity. They may be lower for intentionally roughened materials, down to 0 for textured carbon. They may be up to 2 for extremely smooth surfaces. The modified Vaughan parameter $E_{\text {threshold }}$ and Vaughan rugosity parameters were also fitted on $0-300 \mathrm{eV}$ and $0^{\circ}-40^{\circ}, k_{E}$ and $k_{\theta}$ being constrained between 0.5 and 2 .

Fil et al. studied the sensitivity to surfaces' TEEY curves of the multipactor threshold obtained by simulation $^{49}$. They realised more than four hundred multipactor simulations with the software Spark $3 \mathrm{D}^{50}$. The simulated geometry was a waveguide of width $9.525 \mathrm{~mm}$ and a gap of $0.1 \mathrm{~mm}$, working at $12 \mathrm{GHz}$. They showed that, for this system, the calculated multipactor threshold was particularly sensitive to the value of $E_{c 1}$ and to the profile of $\sigma\left(E_{c 1}<E_{0}<E_{0, \max }\right)$. $E_{0 \text {, max }}$ is the energy for which the TEEY is maximum and is generally between $200 \mathrm{eV}$ and $1 \mathrm{keV}$. Very few electrons impact surfaces with energies this high, and multipactor apparition is probably most influenced by the profile of $\sigma$ between $E_{c 1}$ and $E_{c 1}$ plus a few tenths of eV. POTOMAC simulations showed that the $E_{0}$ and $\theta_{0}$ validity range defined in Section II were still relevant in this geometry.

These requirements were shown to be valid in one particular geometry only, and did not address incidence angle problematics. In order to compare Dionne's and Vaughan's models in a way that is not relevant only for multipactor in small gap waveguides, we decided to evaluate their performance on more criteria. For $\theta_{0}$ in $0^{\circ}-40^{\circ}$, we calculated the standard deviation between the measured and calculated TEEY for $E_{0}<E_{c 1}$ and for $E_{0}>E_{c 1}$; the standard deviation on the whole energy range was also computed. Finally, we calculated the standard deviation between the measured and calculated first cross-over energies. Although this approach does not quantify the contribution of backscattered electrons to multipactor, it is a convenient way to compare the different models to the measures.

\section{Sample $\mathrm{Cu} \# 1$ results}

The measured and fitted TEEY for the sample $\mathrm{Cu} \# 1$ after heating are represented in Fig. 4. The standard deviations between the models and the measurements are presented in Tab. II. Results show an overall good agreement between both models and measurements, on the validity range we defined. For low energies under $E_{c 1}$ and for all $\theta_{0}$, Dionne's model underestimates the TEEY and Vaughan's model fits better. On the contrary, Dionne's model show better agreement than Vaughan's for energies above $E_{c 1}-$ excluding $\theta_{0}=60^{\circ}$. On the whole energy range, Vaughan's model shows a smaller standard deviation from the measurements than Dionne's model at $\theta_{0}=0^{\circ}$, but it rapidly grows with $\theta_{0}$ and Dionne fits better for $40^{\circ}$ and $60^{\circ}$. Dionne's error is more independent from the incidence angle than Vaughan's. As the modified Vaughan model was designed to fit $E_{c 1}\left(0^{\circ}\right)$, it's deviation from the measurements at this point is very low. It increases with $\theta_{0}$ and, at $60^{\circ}$, Dionne fits better than Vaughan.

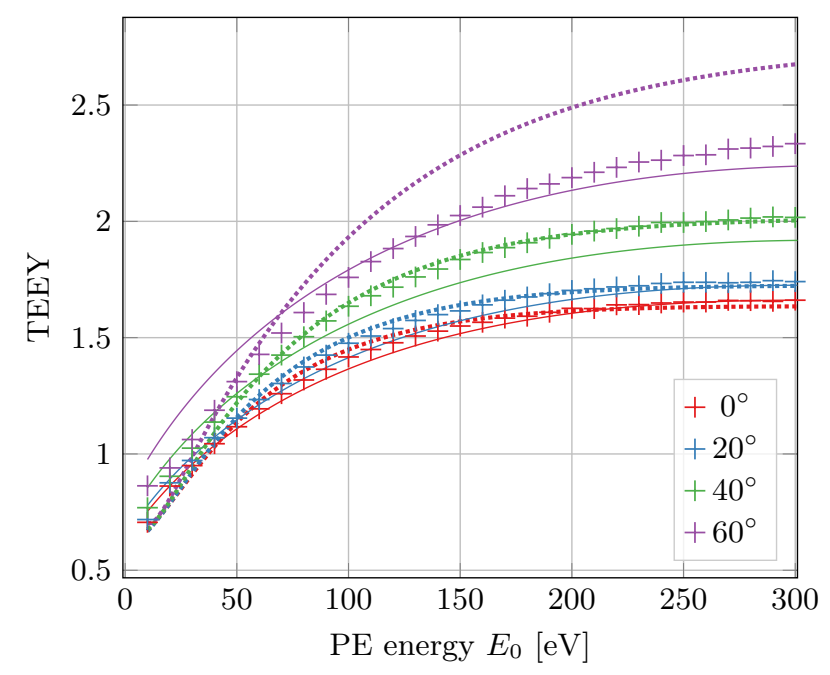

FIG. 4: Measured and fitted TEEY on $\mathrm{Cu} \# 1$ (heated; + measurements, ....... Dionne fit, _ Vaughan fit) [Associated dataset available at

http://dx.doi.org/10.5281/zenodo.3648704]

(Ref. 44)

The results for eroded $\mathrm{Cu} \# 1$ are represented in Fig. 5, and standard deviations in Tab. III. Excluding $\theta_{0}=20^{\circ}$, results are similar to heated $\mathrm{Cu} \# 1$. Vaughan fits better $\sigma$ 


\begin{tabular}{cccccc}
\hline & & $\sigma\left(E_{0}<E_{c 1}\right)$ & $E_{c 1}[\mathrm{eV}]$ & $\sigma\left(E_{0}>E_{c 1}\right)$ & $\sigma(0-300 \mathrm{eV})$ \\
\hline Dionne & $0^{\circ}$ & 0.054 & 1.9 & $\mathbf{0 . 0 2 5}$ & 0.029 \\
& $20^{\circ}$ & 0.066 & 3.1 & $\mathbf{0 . 0 2 0}$ & $\mathbf{0 . 0 2 8}$ \\
& $40^{\circ}$ & 0.101 & $\mathbf{5 . 7}$ & $\mathbf{0 . 0 2 3}$ & $\mathbf{0 . 0 3 4}$ \\
Vaughan & $0^{\circ}$ & $\mathbf{0 . 0 2 7}$ & $\mathbf{0 . 0}$ & 0.026 & $\mathbf{0 . 0 2 6}$ \\
& $20^{\circ}$ & $\mathbf{0 . 0 3 7}$ & $\mathbf{1 . 6}$ & 0.038 & 0.038 \\
& $40^{\circ}$ & $\mathbf{0 . 0 8 0}$ & 6.1 & 0.086 & 0.086 \\
\hline
\end{tabular}

TABLE II: Heated $\mathrm{Cu} \# 1$ standard deviation for the different regions of interest. The lowest standard deviations are given in bold.

under $E_{c 1}$, and Dionne fits better after $E_{c 1}$. On the whole energy range, the error of Dionne is more constant than Vaughan's. Again, the Vaughan model perfectly matches $E_{c 1}\left(0^{\circ}\right)$. Here, $\sigma\left(E_{0}, 0^{\circ}\right)>\sigma\left(E_{0}, 20^{\circ}\right)$, which cannot be reproduced by Dionne nor Vaughan. Plus, the TEEY drastically increases at $60^{\circ}$ and is thus underestimated by the models. Vaughan results are similar to Dionne.

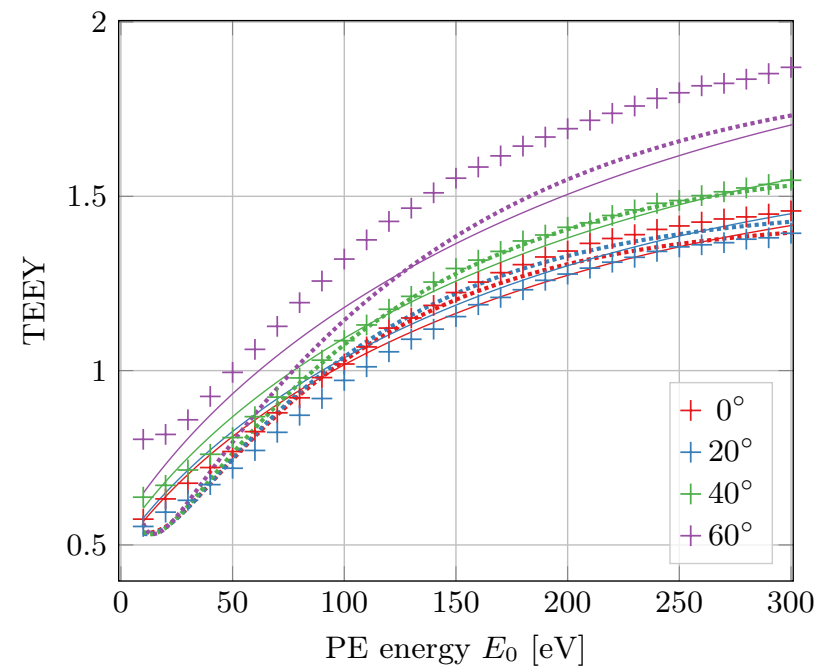

FIG. 5: Measured and fitted TEEY on $\mathrm{Cu} \# 1$ (eroded; + measurements, ....... Dionne fit, _ Vaughan fit) Associated dataset available at http://dx.doi.org/10.5281/zenodo.3648704]

(Ref. 44)

The obtained fitting parameters for $\mathrm{Cu} \# 1$ are summarized in Tab. IV.

\section{E. Sample $\mathrm{Cu} \# 2$ results}

The results for the sample $\mathrm{Cu} \# 2$ without any surface treatment are represented in Fig. 6 and the standard errors in Tab. V. Both Dionne and Vaughan models work very well. Dionne fits better $\sigma$ on the whole energy range and for all angles - except for $\sigma\left(E 0>E_{c 1}, \theta_{0}=40^{\circ}\right)$. Dionne even fits $60^{\circ}$ which is out of the validity range.

\begin{tabular}{|c|c|c|c|c|c|}
\hline & & $\sigma\left(E_{0}<E_{c 1}\right)$ & $E_{c 1}[\mathrm{eV}]$ & $\sigma\left(E_{0}>E_{c 1}\right)$ & $\sigma(0-300 \mathrm{eV})$ \\
\hline \multirow[t]{3}{*}{ Dionne } & $0^{\circ}$ & 0.042 & 1.3 & 0.040 & 0.041 \\
\hline & $20^{\circ}$ & 0.047 & 15.3 & 0.054 & 0.052 \\
\hline & $40^{\circ}$ & 0.075 & 2.5 & 0.010 & 0.040 \\
\hline \multirow[t]{3}{*}{ Vaughan } & $0^{\circ}$ & 0.026 & 0.0 & 0.057 & 0.050 \\
\hline & $20^{\circ}$ & 0.082 & 16.6 & 0.038 & 0.057 \\
\hline & $40^{\circ}$ & 0.036 & 4.9 & 0.026 & 0.029 \\
\hline
\end{tabular}

TABLE III: Eroded $\mathrm{Cu} \# 1$ standard deviation for the different regions of interest. The lowest standard deviations are given in bold.

\begin{tabular}{llcc}
\hline & & Heated & Eroded \\
\hline Dionne & $A$ & 6.22 & 5.43 \\
& $n$ & 1.00 & 1.00 \\
& $d[\mathrm{~nm}]$ & 7.76 & 17.42 \\
$S$ & 0.13 & 0.06 \\
& $k_{a}$ & 0.92 & 0.74 \\
& $k_{b}\left[^{\circ}\right]$ & 2.26 & 0.00 \\
& $\Phi[\mathrm{eV}]$ & 4.65 & - \\
& $\eta_{i}$ & 0.25 & - \\
Vaughan & $\sigma_{\max }$ & 1.66 & 1.53 \\
& $E_{0, \max }[\mathrm{eV}]$ & 300 & 550 \\
& $E_{\text {threshold }}[\mathrm{eV}]$ & -21.81 & -28.93 \\
& $k_{E}$ & 0.50 & 1.72 \\
& $k_{\theta}$ & 2.00 & 2.00 \\
\hline
\end{tabular}

TABLE IV: Evolution of $\mathrm{Cu} \# 1$ fitting parameters

Meanwhile, Vaughan fits better $E_{c 1}$.

\begin{tabular}{clcccc}
\hline & & $\sigma\left(E_{0}<E_{c 1}\right)$ & $E_{c 1}[\mathrm{eV}]$ & $\sigma\left(E_{0}>E_{c 1}\right)$ & $\sigma(0-300 \mathrm{eV})$ \\
\hline Dionne & $0^{\circ}$ & $\mathbf{0 . 0 0 7}$ & 3.2 & $\mathbf{0 . 0 2 6}$ & $\mathbf{0 . 0 2 5}$ \\
& $20^{\circ}$ & $\mathbf{0 . 0 0 2}$ & 2.1 & $\mathbf{0 . 0 3 1}$ & $\mathbf{0 . 0 3 0}$ \\
& $40^{\circ}$ & $\mathbf{0 . 0 8 0}$ & 3.6 & 0.023 & $\mathbf{0 . 0 3 0}$ \\
Vaughan & $0^{\circ}$ & 0.102 & $\mathbf{0 . 0}$ & 0.038 & 0.045 \\
& $20^{\circ}$ & 0.113 & $\mathbf{0 . 8}$ & 0.043 & 0.051 \\
& $40^{\circ}$ & 0.200 & $\mathbf{1 . 3}$ & $\mathbf{0 . 0 2 0}$ & 0.055 \\
\hline
\end{tabular}

TABLE V: As received $\mathrm{Cu} \# 2$ standard deviation for the different regions of interest. The lowest standard deviations are given in bold.

Heated $\mathrm{Cu} \# 2$ results are represented in Fig. 7, standard errors in Tab. VI. For this material, the Dionne model fits much better the measurements on all the regions of interest, as Vaughan underestimates the TEEY. However, Vaughan matches better $E_{c 1}\left(0^{\circ}\right)$. Here, $\sigma\left(60^{\circ}\right)>\sigma\left(40^{\circ}\right)$, leading to a wild overestimation of the TEEY at $60^{\circ}$ for both models.

Finally, the measured and fitted TEEY of eroded $\mathrm{Cu} \# 2$ are represented in Fig. 8 and the standard deviations in Tab. VII. According to Fil et al. criteria $^{49}$ and considering only $\theta_{0}=0^{\circ}$, Vaughan model is more 


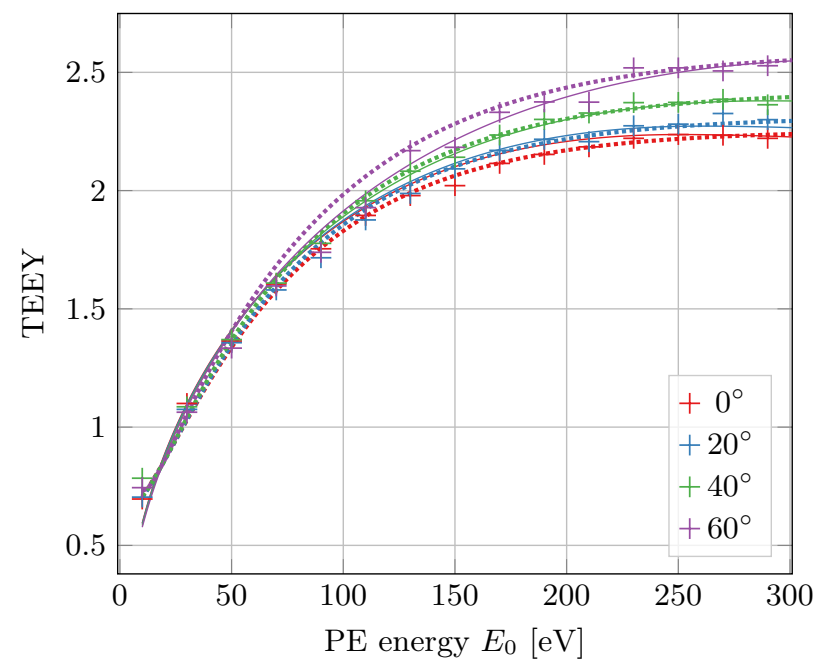

FIG. 6: Measured and fitted TEEY on $\mathrm{Cu} \# 2$ (as received; + measurements, ....... Dionne fit, Vaughan fit) [Associated dataset available at http://dx.doi.org/10.5281/zenodo.3648704]

(Ref. 44)

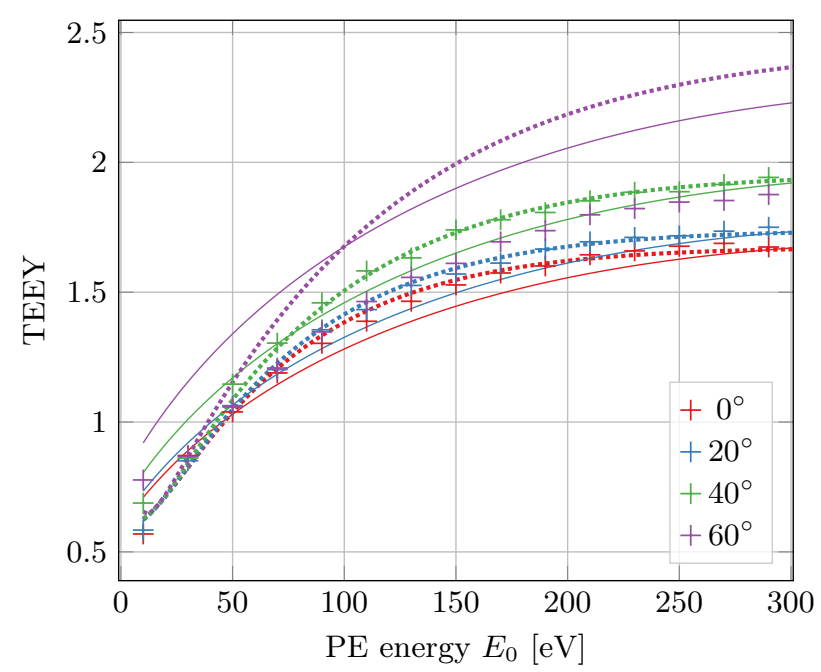

FIG. 7: Measured and fitted TEEY on $\mathrm{Cu} \# 2$ (heated; + measurements, ....... Dionne fit, __ Vaughan fit) [Associated dataset available at http://dx.doi.org/10.5281/zenodo.3648704] (Ref. 44)

appropriate for multipactor simulations. However, for $\theta_{0}$ in $0^{\circ}-40^{\circ}$, the standard deviation of Dionne is lower for $\sigma(0-300 \mathrm{eV})$ and $\sigma\left(E_{0}<E_{c 1}\right)$. For $\theta_{0} \geq 20^{\circ}$, Dionne also fits better $\sigma\left(E_{0}>E_{c 1}\right)$. The measured TEEY at $60^{\circ}$ is lower than the TEEY at $40^{\circ}$, leading to a wide overestimation of the measurements by both models.

All fitting parameters are listed in Tab. VIII.

\begin{tabular}{|c|c|c|c|c|c|}
\hline & & $\sigma\left(E_{0}<E_{c 1}\right)$ & $E_{c 1}[\mathrm{eV}]$ & $\sigma\left(E_{0}>E_{c 1}\right)$ & $\sigma(0-300 \mathrm{eV})$ \\
\hline \multirow[t]{3}{*}{ Dionne } & $0^{\circ}$ & 0.052 & 0.9 & 0.022 & 0.028 \\
\hline & $20^{\circ}$ & 0.033 & 1.0 & 0.017 & 0.020 \\
\hline & $40^{\circ}$ & 0.044 & 2.9 & 0.023 & 0.027 \\
\hline \multirow[t]{3}{*}{ Vaughan } & $0^{\circ}$ & 0.101 & 0.1 & 0.060 & 0.067 \\
\hline & $20^{\circ}$ & 0.117 & 3.7 & 0.056 & 0.068 \\
\hline & $40^{\circ}$ & 0.134 & 11.0 & 0.051 & 0.068 \\
\hline
\end{tabular}

TABLE VI: Heated $\mathrm{Cu} \# 2$ standard deviation for the different regions of interest. The lowest standard deviations are given in bold.

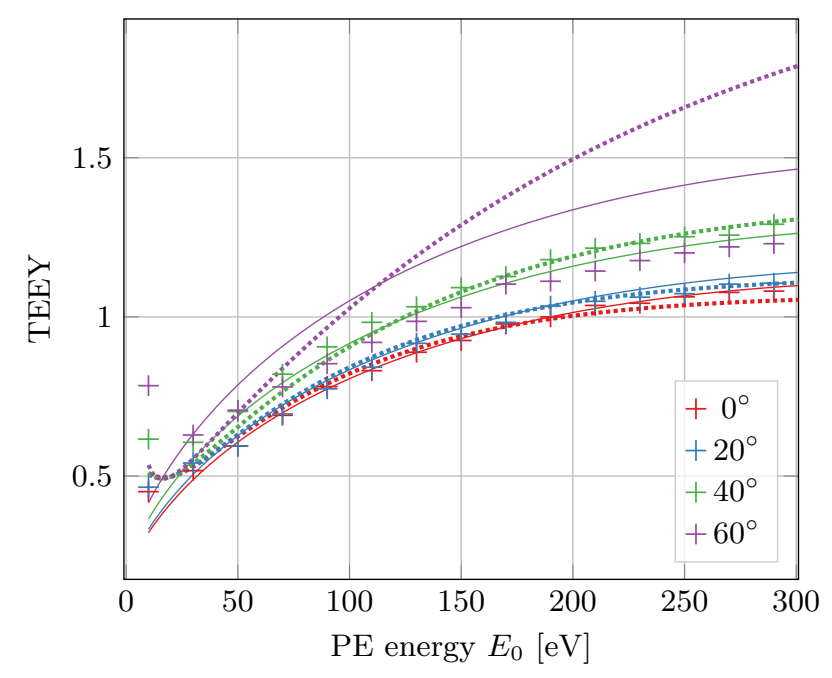

FIG. 8: Measured and fitted TEEY on $\mathrm{Cu} \# 2$ (eroded; + measurements, ....... Dionne fit, __ Vaughan fit) [Associated dataset available at http://dx.doi.org/10.5281/zenodo.3648704] (Ref. 44)

\section{DISCUSSION}

The proposed model works very well on the defined validity range (i.e., $E_{0}$ in $0-300 \mathrm{eV}$ and $\theta_{0}$ in $0^{\circ}-40^{\circ}$ ).

According to Fil et $a .^{49}$, TEEY models must fit precisely $\sigma\left(E_{0}>E_{c 1}\right)$ to ensure a precise prediction of the

\begin{tabular}{cccccc}
\hline & & $\sigma\left(E_{0}<E_{c 1}\right)$ & $E_{c 1}[\mathrm{eV}]$ & $\sigma\left(E_{0}>E_{c 1}\right)$ & $\sigma(0-300 \mathrm{eV})$ \\
\hline Dionne & $0^{\circ}$ & $\mathbf{0 . 0 2 4}$ & 6.4 & 0.024 & $\mathbf{0 . 0 2 5}$ \\
& $20^{\circ}$ & $\mathbf{0 . 0 2 9}$ & 10.9 & $\mathbf{0 . 0 0 8}$ & $\mathbf{0 . 0 2 3}$ \\
Vaughan & $0^{\circ}$ & $\mathbf{0 . 0 5 9}$ & $\mathbf{6 . 7}$ & $\mathbf{0 . 0 1 2}$ & $\mathbf{0 . 0 3 9}$ \\
& 0.042 & $\mathbf{0 . 1}$ & $\mathbf{0 . 0 0 7}$ & 0.035 \\
& $20^{\circ}$ & 0.050 & $\mathbf{8 . 4}$ & 0.023 & 0.041 \\
& $40^{\circ}$ & 0.107 & 9.2 & 0.030 & 0.072 \\
\hline
\end{tabular}

TABLE VII: Eroded $\mathrm{Cu} \# 2$ standard deviation for the different regions of interest. The lowest standard deviations are given in bold. 


\begin{tabular}{llccc}
\hline & & As received & Heated & Eroded \\
\hline Dionne & $A$ & 6.67 & 4.98 & 8.50 \\
& $n$ & 1.00 & 1.00 & 1.00 \\
& $d[\mathrm{~nm}]$ & 9.47 & 12.40 & 9.84 \\
$S$ & 0.14 & 0.10 & 0.04 \\
& $k_{a}$ & 0.40 & 0.83 & 1.13 \\
& $k_{b}\left[^{\circ}\right]$ & 8.04 & 1.12 & 0.00 \\
& $\Phi[\mathrm{eV}]$ & 4.65 & - & - \\
& $\eta_{i}$ & 0.25 & - & - \\
Vaughan & $\sigma_{\max }$ & 2.24 & 1.70 & 1.12 \\
& $E_{0, \max }[\mathrm{eV}]$ & 250 & 390 & 390 \\
& $E_{\text {threshold }}[\mathrm{eV}]$ & 1.11 & -25.09 & -6.51 \\
& $k_{E}$ & 2.00 & 0.50 & 0.50 \\
& $k_{\theta}$ & 0.82 & 2.00 & 2.00 \\
\hline
\end{tabular}

TABLE VIII: Evolution of $\mathrm{Cu} \# 2$ fitting parameters

multipactor threshold. This conclusion is valid only in small gaps structures. The Dionne model address this requirement on more materials and incidence angles than the Vaughan model. However, the second most important requirement is the precision around $E_{c 1}$, which is better answered by Vaughan's model. Still, as Dionne's model can discriminate secondary from backscattered electrons, we think that it is more suitable for multipactor simulations.

On a more general point of view, the standard deviation of $\sigma(0-300 \mathrm{eV})$ is generally lower for Dionne than for Vaughan. For $\mathrm{Cu} \# 2$, it is always the case. For $\mathrm{Cu} \# 1$, this difference is less significant. Dionne standard deviation of $\sigma\left(E_{0}<E_{c 1}\right)$ was lower for $\mathrm{Cu} \# 2$, but higher for $\mathrm{Cu} \# 1$.

It is to be noted that Vaughan's fit was much better than Dionne for energies higher than a few hundreds of $\mathrm{eV}$, and that it's implementation is easier. Vaughan rugosity parameters are often inconsistent, for example for heated $\mathrm{Cu} \# 2$, where $k_{E}=0.50$ suggests that the sample is very rough, while $k_{\theta}=2.00$ tells the exact opposite.

Results however show some discrepancy in the very low energy range $(\lesssim 20 \mathrm{eV})$, especially for non-normal incidences and eroded materials. We propose several explanations; firstly, the range expression (Eq. (5)) was obtained with the hypothesis that electrons lost their energy in the material in a continuous way (continuous slowing down approximation), which is inexact at low energies. In reality, the range may be constant below a few tenths of $\mathrm{eV}^{51,52}$. Secondly, we set a constant IBEEY as we lack literature on the subject, which is a very important hypothesis. Thirst, the potential of the sample holder $(-9 \mathrm{~V}$ for these experiments) can deflect significantly very low-energy electrons at non-normal incidence. A method that polarizes the collector instead of the sample has been developed ${ }^{53}$. Finally, the magnetic field of the Earth may have an influence for very low energies ${ }^{53}$.

In Fig. 9, we represented $\theta_{\text {equ }}$ as a function of $\theta_{0}$ (Eq. (8)) for the different materials, with the values of $k_{a}$ and $k_{b}$ listed in Tab. IV and VIII. We also plotted Zhang et al. results, obtained with Monte-Carlo simulations; the three black lines represent the evolution of the equivalent angle for three copper samples with different random rugosity profiles ${ }^{42}$. As we can see from the shape of Zhang's curves, the choice of modelling the $\theta_{\text {equ }}$ law with a firstorder polynomial seems to be justified. However, the materials studied in this paper are all softer than Zhang's.

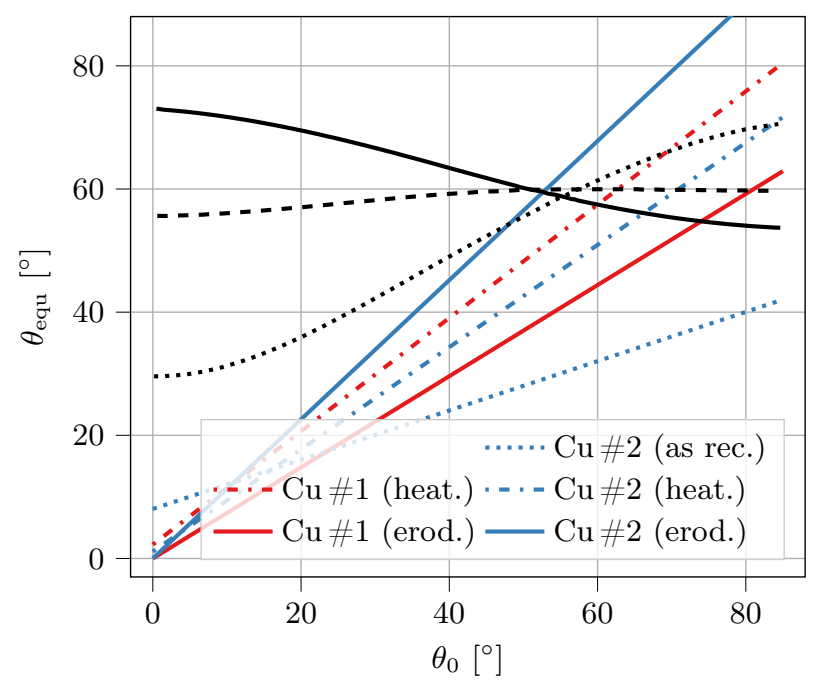

FIG. 9: $\theta_{\text {equ }}$ laws for different materials; black lines represent Zhang et al. simulation results on different random surfaces with a Gaussian height profile $^{42}$

All trends are not faithfully reproduced when increasing $\theta_{0}$ leads to a reduction of $\sigma$ (i.e. $\mathrm{Cu} \# 1$ eroded, $\mathrm{Cu} \# 2$ heated, $\mathrm{Cu} \# 2$ eroded). In these cases, the variation of $\sigma$ with $\theta_{0}$ is non monotonous, which cannot be reproduced by Eq. (8). Using higher-order polynomials of the form $\theta_{\text {equ }}=\sum k_{i} \theta_{0}^{i}$ may solve the issue. However, the values of the coefficients $k_{i}$ have to be chosen carefully so that, for the defined range of $\theta_{0}, \theta_{\text {equ }} \in[0, \pi]$. We noted that, even for polynomials of order two respecting this condition, $\theta_{\text {equ }}$ could take very unlikely values for $\theta_{0}>40^{\circ}$.

New emission yield measurements campaigns associated with rugosity profile studies would be required to investigate further the relation between $\theta_{0}$ and $\theta_{\text {equ }}$. Also, the hypothesis of a random rough surface with a Gaussian height distribution may be not representative of the real surface profile.

For the two materials, the surface treatments reduce contamination and thus the emission yield. As SEs have a low energy, the only ones able to escape are those produced in the first nanometers below the surface. Thus, they are very influenced by the presence of contaminants ${ }^{16}$. For both samples, the reduction of the contamination layer reduces the parameter $k_{b}$, which is 0 for eroded coppers. Our interpretation is that, for this materials, the contamination layer at the surface of the samples has a rougher surface state than the bulk material underneath; this is particularly noticeable on 
$\mathrm{Cu} \# 2$, where the difference of rugosity between the sample without any surface treatment and after the heating is tremendous. It is to be noted that, even for clean materials with $k_{a} \approx 1$ and $k_{b} \approx 0$, the introduction of $\theta_{\text {equ }}$ increased the accuracy of our model. We observed that the simpler law $\theta_{\text {equ }}=k \theta_{0}{ }^{13}$ was precise enough for all materials, including $\mathrm{Cu} \# 2$ as received; though, it cannot model the Zhang et al. materials.

The validity of the present model has not been tested yet on dielectrics. As the dielectric material charge would deflect electrons, the angle between the electron canon and the sample $\theta_{\text {canon }}$ would be different from the electrons incidence angle $\theta_{0}$. Proper measurements require additional work and are not in the scope of the present study.

\section{CONCLUSIONS}

In this paper, we developed a simple extension of Dionne's model to three-dimension. We used the refraction law for electrons to compute the range of electrons for various incidence angles. We used the concept of equivalent angle $\theta_{\text {equ }}$ to model the differences between macroscopic and microscopic incidence angle, as well as shading and multigeneration effects.

The present model was compared to in-house measurements on two copper samples with several surface treatments. The fit was made on the low-energy range $(0-300 \mathrm{eV})$ and low-angle range $\left(0^{\circ}-40^{\circ}\right)$, which is relevant for multipactor simulations. As a whole, the model fit experimental data in these ranges. The fit is however less good for high incidence angles and low energies, as well as for some materials where the evolution of $\sigma$ with $\theta_{0}$ is not monotonous.

The introduction of $\theta_{\text {equ }}$ was indispensable for the most contaminated material $(\mathrm{Cu} \# 2$ as received), but also greatly increased the accuracy of the fit for purer materials. The relation between $\theta_{0}$ and $\theta_{\text {equ }}$ should be explored, with Monte-Carlo simulations and emission yield measurements associated with rugosity profile studies. As magnetic fields were shown to influence electron emission from rough samples ${ }^{17}$, studying the link between $\theta_{\text {equ }}$ and magnetic fields would be another step towards extensive multipactor studies in presence of magnetic fields. Fits on silver led to similar results but were not included in this publication.

A comparison was made with modified Vaughan model, widely used in the multipactor community. At very low energy, the Dionne model showed to be more precise. At higher energies up to $300 \mathrm{eV}$, Dionne's model gave similar or better results than Vaughan.

Several points could enhance the model: firstly, it has been proven that SLAB model was too simplistic ${ }^{54}$ and thus could be replaced by OKGM model even if it generally underestimates $\eta_{e}^{55,56}$. A simple and physical model would be of great value, as it would avoid the expensive computational cost of tabulating large arrays. Plus, it would allow a deeper understanding of the physics at stake.

Secondly, the modeling of $\eta_{i}$ is also simplistic - we lack a simple physical model for the IBEEY. Finally, the used range function is not valid on the whole energy range, $R$ being almost constant below a few tenths of $\mathrm{eV}^{51,52}$.

\section{ACKNOWLEDGMENTS}

This work has been carried out within the framework of the EUROfusion Consortium and has received funding from the Euratom research and training programme 2014-2018 and 2019-2020 under grant agreement No 633053. The views and opinions expressed herein do not necessarily reflect those of the European Commission.

${ }^{1}$ J. de Lara, F. Perez, M. Alfonseca, L. Galan, I. Montero, E. Roman, and D. Garcia-Baquero, "Multipactor prediction for onboard spacecraft RF equipment with the MEST software tool," IEEE Transactions on Plasma Science 34, 476-484 (2006).

${ }^{2}$ M. Goniche, C. El Mhari, M. Francisquez, S. Anza Hormigo, J. Belo, P. Hertout, and J. Hillairet, "Modelling of power limit in $\mathrm{RF}$ antenna waveguides operated in the lower hybrid range of frequency," Nuclear Fusion 54, 013003 (2014).

${ }^{3}$ C. Bourat and J.-M. Joly, "On multipactor effect in a $600 \mathrm{MHz}$ RF cavity used in electron linear accelerator," IEEE Transactions on Electrical Insulation 24, 1045-1048 (1989).

${ }^{4}$ E. Sorolla, Contribution to modeling multipactor and corona discharges in high power electromagnetic fields, Ph.D. thesis, École Polytechnique Fédérale de Lausanne (2012).

${ }^{5}$ A. J. Hatch and H. B. Williams, "Multipacting Modes of HighFrequency Gaseous Breakdown," Physical Review 112, 681-685 (1958).

${ }^{6}$ E. F. Vance, "One-Sided Multipactor Discharge Modes," Journal of Applied Physics 34, 3237-3242 (1963).

${ }^{7}$ A. G. Sazontov, V. A. Sazontov, and N. K. Vdovicheva, "Multipactor Breakdown Prediction in a Rectangular Waveguide: Statistical Theory and Simulation Results," Contributions to Plasma Physics 48, 331-346 (2008).

${ }^{8}$ V. E. Semenov, E. I. Rakova, D. Anderson, M. Lisak, and J. Puech, "Multipactor in rectangular waveguides," Physics of Plasmas 14, 033501 (2007).

${ }^{9}$ M. Francisquez, Power Limit Modeling of Lower Hybrid Antenna Waveguides in Tokamaks, Bachelor of arts honors thesis, Dartmouth College (Hanover, NH) (2012).

${ }^{10}$ C. Chang, G. Liu, C. Tang, C. Chen, and J. Fang, "Review of recent theories and experiments for improving high-power microwave window breakdown thresholds," Physics of Plasmas $\mathbf{1 8}$ (2011), 10.1063/1.3560599.

${ }^{11}$ E. Sorolla, M. Belhaj, J. Sombrin, and J. Puech, "New multipactor dynamics in presence of dielectrics," Physics of Plasmas 24, 103508 (2017).

${ }^{12}$ A. Plaçais, E. Sorolla, M. Belhaj, J. Hillairet, and J. Puech, "Influence of work function on the multipactor threshold," in 2018 IEEE MTT-S International Conference on Numerical Electromagnetic and Multiphysics Modeling and Optimization (NEMO) (IEEE, Reykjavik, Iceland, 2018) pp. 1-4.

${ }^{13}$ A. Plaçais, M. Belhaj, J. Hillairet, and J. Puech, "POTOMAC: Towards a Realistic Secondary and Backscattered Emission Model for the Multipactor," in 2019 IEEE Pulsed Power \&6 Plasma Science (PPPS) (IEEE, Orlando, USA, 2019) pp. 1-4.

${ }^{14}$ A. Dekker, "Secondary Electron Emission," in Solid State Physics, Vol. Volume 6 (1958) pp. 251-311.

${ }^{15} \mathrm{~K}$. Nishimura, T. Itotani, and K. Ohya, "Influence of Surface Roughness on Secondary Electron Emission and Electron 
Backscattering from Metal Surface," Japanese Journal of Applied Physics 33, 4727-4734 (1994).

${ }^{16}$ T. Gineste, M. Belhaj, G. Teyssedre, and J. Puech, "Investigation of the electron emission properties of silver: From exposed to ambient atmosphere Ag surface to ion-cleaned Ag surface," Applied Surface Science 359, 398-404 (2015).

${ }^{17}$ N. Fil, M. Belhaj, J. Hillairet, J. Puech, and R. Mathevet, "Electron emission under uniform magnetic field of materials for fusion and space applications," Fusion Engineering and Design (2017), 10.1016/j.fusengdes.2017.03.039.

${ }^{18}$ M. A. Furman and M. T. F. Pivi, "Probabilistic model for the simulation of secondary electron emission," Physical Review Special Topics - Accelerators and Beams 5, 124404 (2002).

${ }^{19}$ M. A. Furman and M. T. F. Pivi, "Erratum: Probabilistic model for the simulation of secondary electron emission [Phys. Rev. ST Accel. Beams 5 , 124404 (2002)]," Physical Review Special Topics - Accelerators and Beams 16, 069901 (2013).

${ }^{20}$ D. Sydorenko, Particle-in-Cell Simulations of Electron Dynamics in Low Pressure Discharges with Magnetic Fields, Ph.D. thesis, University of Saskatchewan, Canada (2006).

${ }^{21}$ G. F. Dionne, "Effects of secondary electron scattering on secondary emission yield curves," Journal of Applied Physics 44, 5361-5364 (1973).

${ }^{22}$ N. Balcon, M. Belhaj, T. Tondu, J. C. Mateo-Velez, and D. Payan, "Secondary electron emission of cover glasses: Temperature and incident flux effects," in International Symposium on Materials in the Space Environment, edited by L. Ouwehand (ESA Communications, Noordwijk, The Netherlands, 2013).

${ }^{23}$ J. Vaughan, "A new formula for secondary emission yield," IEEE Transactions on Electron Devices 36, 1963-1967 (1989).

${ }^{24}$ J. Vaughan, "Secondary emission formulas," IEEE Transactions on Electron Devices 40, 830 (1993).

${ }^{25}$ S. Barral, K. Makowski, Z. Peradzyński, N. Gascon, and M. Dudeck, "Wall material effects in stationary plasma thrusters. II. Near-wall and in-wall conductivity," Physics of Plasmas 10, 4137-4152 (2003).

${ }^{26}$ R. Seviour, "The Role of Elastic and Inelastic Electron Reflection in Multipactor Discharges," IEEE Transactions on Electron Devices 52, 1927-1930 (2005).

${ }^{27}$ M. S. Feldman, A. A. Hubble, R. Spektor, and P. T. Partridge, "Effects of Backscattered Electrons on Multipactor Simulations with Parallel Magnetic Fields," in 2018 IEEE MTT-S International Conference on Numerical Electromagnetic and Multiphysics Modeling and Optimization (NEMO) (IEEE, Reykjavik, Iceland, 2018) pp. 1-3.

${ }^{28}$ M. Villemant, P. Sarrailh, M. Belhaj, C. Inguimbert, and L. Garrigues, "Modélisation de l'émission secondaire dans les propulseurs à courant de Hall et les applications RF," Tech. Rep. (ONERA, Toulouse, France, 2017).

${ }^{29}$ A. Jablonski, P. Mrozek, G. Gergely, M. Menhyárd, and A. Sulyok, "The inelastic mean free path of electrons in some semiconductor compounds and metals," Surface and Interface Analysis 6, 291-294 (1984).

${ }^{30} \mathrm{~J}$. Greenwood, "The correct and incorrect generation of a cosine distribution of scattered particles for Monte-Carlo modelling of vacuum systems," Vacuum 67, 217-222 (2002).

${ }^{31}$ M. S. Chung and T. E. Everhart, "Simple calculation of energy distribution of low-energy secondary electrons emitted from metals under electron bombardment," Journal of Applied Physics 45, 707-709 (1974).

${ }^{32}$ R. Cimino, L. A. Gonzalez, R. Larciprete, A. Di Gaspare, G. Iadarola, and G. Rumolo, "Detailed investigation of the low energy secondary electron yield of technical $\mathrm{Cu}$ and its relevance for the LHC," Physical Review Special Topics - Accelerators and Beams 18, 051002 (2015).

${ }^{33}$ C. P. Vicente, M. Mattes, D. Wolk, B. Mottet, H. Hartnagel, J. Mosig, and D. Raboso, "Multipactor breakdown prediction in rectangular waveguide based components," in IEEE MTT$S$ International Microwave Symposium Digest, 2005., Vol. 2005 (IEEE, 2005) pp. 1055-1058.
${ }^{34}$ N. Fil, M. Belhaj, J. Hillairet, and J. Puech, "Multipactor threshold sensitivity to Total Electron Emission Yield in parallel-plate waveguide and TEEY models accuracy," in 2016 IEEE MTT$S$ International Microwave Symposium (IMS), Vol. 23 (IEEE, 2016) pp. 1-4.

${ }^{35}$ J. Cazaux, "Calculated influence of work function on SE escape probability and Secondary Electron Emission yield," Applied Surface Science 257, 1002-1009 (2010).

${ }^{36}$ G. F. Dionne, "Origin of secondary-electron-emission yield-curve parameters," Journal of Applied Physics 46, 3347-3351 (1975).

37 J. R. Young, "Penetration of Electrons and Ions in Aluminum," Journal of Applied Physics 27, 1-4 (1956).

${ }^{38}$ N. D. Lang and W. Kohn, "Theory of Metal Surfaces: Work Function," Physical Review B 3, 1215-1223 (1971).

${ }^{39}$ M. Sastry, "On the correlation between the inelastic mean free paths of secondary electrons and the secondary-electron yield parameter in some metal films," Journal of Electron Spectroscopy and Related Phenomena 106, 93-99 (2000).

${ }^{40}$ K. I. Grais and A. M. Bastawros, "A study of secondary electron emission in insulators and semiconductors," Journal of Applied Physics 53, 5239-5242 (1982).

${ }^{41}$ C. Weisbuch, H. Benisty, D. Labilloy, R. Houdré, R. P. Stanley, and M. Ilegems, Confined Electrons and Photons, edited by E. Burstein and C. Weisbuch, NATO ASI Series, Vol. 340 (Springer US, Boston, MA, 1995) pp. 288-289.

${ }^{42}$ N. Zhang, M. Cao, W.-Z. Cui, and T.-C. Hu, "Effect of rough surface morphology on secondary electron emission from metal surface," Japanese Journal of Applied Physics 56, 075802 (2017).

${ }^{43}$ V. Baglin, Y. Bozhko, O. Gröbner, B. Henrist, N. Hilleret, C. Scheuerlein, and M. Taborelli, "The Secondary Electron Yield of Technical Material and Its Variation With Surface Treatments," in EPAC 2000 (Vienna, 2000) pp. 217-221.

${ }^{44}$ A. Plaçais, M. Belhaj, and S. Dadouch, "ONERA copper TEEY measurements with various surface state and incidence angle," Zenodo (2020), 10.5281/zenodo.3648704.

${ }^{45}$ D. Drouin, A. R. Couture, D. Joly, X. Tastet, V. Aimez, and R. Gauvin, "CASINO V2.42-A Fast and Easy-to-use Modeling Tool for Scanning Electron Microscopy and Microanalysis Users," Scanning 29, 92-101 (2007).

${ }^{46}$ F. Salvat, A. Jablonski, and C. J. Powell, "ELSEPA-Dirac partial-wave calculation of elastic scattering of electrons and positrons by atoms, positive ions and molecules," Computer Physics Communications 165, 157-190 (2005).

${ }^{47}$ J. R. Lowney, M. T. Postek, Jr., and A. E. Vladar, "Monte Carlo model for SEM linewidth metrology," in Integrated Circuit Metrology, Inspection, and Process Control VIII, Vol. 2196, edited by M. H. Bennett (San Jose, CA, 1994) pp. 85-96.

${ }^{48}$ D. C. Joy and S. Luo, "An empirical stopping power relationship for low-energy electrons," Scanning 11, 176-180 (1989).

${ }^{49}$ N. Fil, M. Belhaj, J. Hillairet, and J. Puech, "Multipactor threshold sensitivity to total electron emission yield in small gap waveguide structure and TEEY models accuracy," Physics of Plasmas 23, 123118 (2016).

${ }^{50}$ Aurorasat, "SPARK3D User Manual," (2015).

${ }^{51} \mathrm{G}$. Wilson and J. R. Dennison, "Approximation of range in materials as a function of incident electron energy," IEEE Transactions on Plasma Science 40, 291-297 (2012).

${ }^{52}$ C. Inguimbert, J. Pierron, M. Belhaj, and J. Puech, "Extrapolated Range Expression for Electrons Down to $10 \mathrm{eV}$," in NSREC (Portland, USA, 2016).

${ }^{53}$ T. Tondu, M. Belhaj, and V. Inguimbert, "Methods for measurement of electron emission yield under low energy electronirradiation by collector method and Kelvin probe method," Journal of Vacuum Science \& Technology A: Vacuum, Surfaces, and Films 28, 1122-1125 (2010).

${ }^{54}$ A. Jablonski, "Elastic backscattering of electrons from surfaces," Surface Science 151, 166-182 (1985).

${ }^{55}$ R. Oswald, E. Kasper, and K. Gaukler, "A multiple scattering theory of elastic electron backscattering from amorphous surfaces," Journal of Electron Spectroscopy and Related Phenomena 
61, 251-274 (1993).

(2014).

${ }^{56}$ A. Jablonski, "Angular distribution of elastic electron backscattering from surfaces: determination of the electron inelastic mean free path," Journal of Physics D: Applied Physics 47, 055301 